\title{
Channel selection for Selective Sensing using Cognitive Radio Nodes
}

\author{
R. Kaniezhil \\ Full-Time Ph.D Research \\ Scholar \\ Dept. of Computer Science \\ Periyar University \\ Salem, Tamilnadu
}

\author{
C. Chandrasekar \\ Associate Professor \\ Dept. of Computer Science \\ Periyar University \\ Salem, Tamilnadu
}

\author{
S. Nithya Rekha \\ Full-Time Ph.D Research \\ Scholar \\ Dept. of Computer Science \\ Periyar University \\ Salem, Tamilnadu
}

\begin{abstract}
In the proposed work, if one service provider is found to be overloaded, it utilizes the spectrum of the other service provider which is found to be underutilized. Thus, CR nodes senses the best available channel for sharing and it provides the list of available channels to the overloaded service provider to utilize the under utilized spectrum efficiently. Spectrum sharing among service providers improve the spectral efficiency, probability efficiency of sensing and reduces the call blockage. If the number of service providers are increased to share the spectrum, this would reduces the high traffic patterns of the calls.
\end{abstract}

\section{General Terms}

Spectrum sharing, Experimental Results.

\section{Keywords}

$\mathrm{CR}$, dynamic spectrum allocation, spectrum sharing, spectrum scarcity, spectrum access, utility performance.

\section{INTRODUCTION}

Traditional wireless networks have predominantly used direct point-to-point or point-to-multipoint (e.g., cellular) topologies. In contrast to conventional point-to-point communications, cooperative communications and networking allows different users or nodes in a wireless network to share resources and to create collaboration through distributed transmission/processing, in which each user's information is sent out not only by the user but also by the collaborating users [2].

Cooperative communications and networking is a new communication paradigm that promises significant capacity and multiplexing gain increase in wireless networks [3], [4]. To solve the problem of spectrum scarcity and spectrum underutilization, the use of CR technology is being considered because of its ability to rapidly and autonomously adapt operating parameters to changing requirements and conditions.

Cognitive radio will improve spectrum utilization in wireless communications systems while accommodating the increasing number of services and applications in wireless networks. A cognitive radio transceiver is able to adapt to the dynamic radio environment and the network parameters to maximize the utilization of the limited radio resources while providing flexibility in wireless access.

Spectrum Sharing (SS) is seen as one possible approach to improving efficiency; intelligent techniques and increased collaboration among networks and terminals are expected to yield benefits in the usage of spectrum. CR has been identified as a possible enabling technique to supply the functionality necessary to achieve flexible and intelligent usage of spectrum, according to the needs of users thus maximizing the opportunities offered by the radio environment.

Due to the immense popularity of mobile phones and wireless LAN systems, high data transmission with a limited frequency band has become a key issue for wireless communication systems. We can in fact dramatically improve the spectrum utilization if we allow a system to utilize dynamically not only a dedicated frequency band, but also the under-utilized frequencies that are allocated to other systems. This concept is referred to as dynamic spectrum sharing.

Spectrum Sharing (SS) as considered by this programme covers both opportunistic sharing, (e.g. sensing of and transmission in spectrum white space) as well as managed sharing of radio resources among multiple operators.

\section{PAPER ORGANIZATION}

The paper structure follows: In Section 2, 3 \& 4, Related work, Cognitive Radio Technology and Channel allocation schemes are briefly introduced. Spectrum sharing is discussed in section 5. Spectrum Sensing Framework for CR Networks and Channel Selection Method for Sensing in section 6 and 7 as problem description. In section 8 and 9 , the performance measures of Spectrum sharing and simulation results are presented. Finally, we draw our conclusions in Section 10.

\section{RELATED WORK}

There have been several research efforts on spectrum sharing in Cognitive radio technologies inorder to inorder to avoid spectrum scarcity and improve the spectrum utilization. This is considered as the main goal of my research work in a coordinated distributed manner and long term spectrum assignment strategy. Many research works were proposed under centralized and decentralized manner of spectrum sharing.

In [14], spectrum utilization is mainly depends upon the percentage of spectrum utilized by one service provider depends on how many users it is serving and how much spectrum each users application demands in a coordinated manner centralized network. In $[15,18]$, the authors proposed a novel spectrum sharing strategy based on the throughput model in a cognitive radio network, where maximum theoretical throughput is taken into account. In [16], the author address the problems of power control and channel assignment jointly. Here, he devised a near-optimal yet simple 
algorithm with linear complexity targeting capacity maximization of a Cognitive radio network while jointly optimizing power and channel allocation among users. In [17], the authors proposed a novel multi-channel connection graph method for achieving a fair spectrum sharing. In [19], spectrum efficiency in multi-hop transmissions is needed by considering route selection and spectrum management.

The differences between this work and previous works are summarized as follows: First of all, resource allocation in a cognitive wireless network is quite different from that in traditional wireless network such as 802.11 based wireless networks due to its special features such as dynamic channel availability, channel heterogeneity and so on (refer to [5] for details. Second, fairness is considered as a major one of this work. However, the proposed work achieves different goals such as minimized active users when there is a high traffic, reduced call blockage, and maximized system efficiency. Third, the paper focuses on the call blocking, channel selection and assignment. However, in most of the previous works on spectrum allocation and sharing $[6,7,8,9,10,11,12]$, allocations are done in unlicensed band in a distributed manner. In this work, spectrum sharing covers with opportunistic spectrum sharing in Inter and Intra network spectrum sharing in a heterogeneous wireless networks.

\section{COGNITIVE RADIO TECHNOLOGY}

The key enabling technology of dynamic spectrum access techniques is cognitive radio (CR) technology, which provides the capability to share the wireless channel with licensed users in an opportunistic manner.

The term, cognitive radio, can formally be defined as follows : A "Cognitive Radio" is a radio that can change its transmitter parameters based on interaction with the environment in which it operates. From this definition, two main characteristics of the cognitive radio can be defined as follows:

- Cognitive capability: Cognitive capability refers to the ability of the radio technology to capture or sense the information from its radio environment. Through this capability, the portions of the spectrum that are unused at a specific time or location can be identified. Consequently, the best spectrum and appropriate operating parameters can be selected.

- Reconfigurability: The cognitive capability provides spectrum awareness whereas reconfigurability enables the radio to be dynamically programmed according to the radio environment.

CR networks are envisioned to provide high bandwidth to mobile users via heterogeneous wireless architectures and dynamic spectrum access techniques. This goal can be realized only through dynamic and efficient spectrum management techniques.

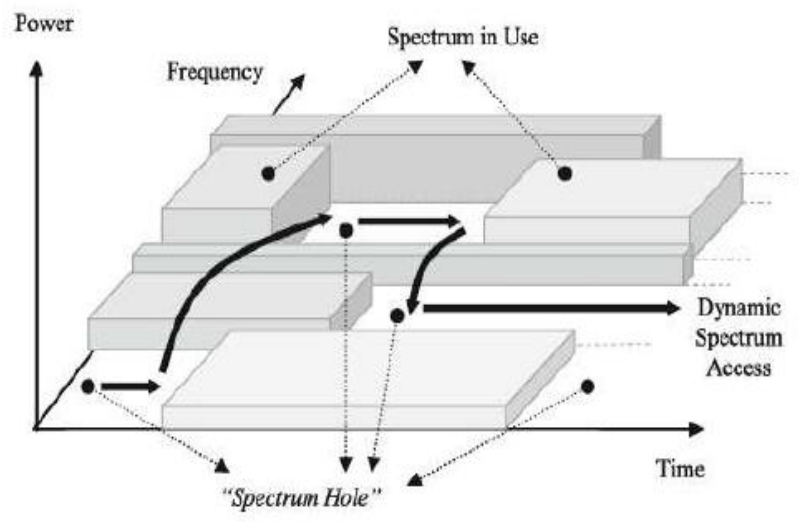

Fig 1: Spectrum Hole Concept

The ultimate objective of the cognitive radio is to obtain the best available spectrum through cognitive capability and reconfigurability as described before. Since most of the spectrum is already assigned, the most important challenge is to share the licensed spectrum without interfering with the transmission of other licensed users as illustrated in Fig 1.

The cognitive radio enables the usage of temporarily unused spectrum, which is referred to as spectrum hole or white space [3]. If this band is further utilized by a licensed user, the cognitive radio moves to another spectrum hole or stays in the same band, altering its transmission power level or modulation scheme to avoid interference as shown in Fig 1.

\subsection{Proposed CR Nodes Sensing}

With the capability of sensing the environment and finding the available spectrum dynamically, CR technique can help to implement spectrum sharing and improve the spectrum utilization efficiency. In the proposed work, CR engines such as learn and decision does the work of Spectrum Sensing. Here, CR nodes helps to provide the list of available channels and it checks the availability of the channels. Mobile nodes get the information of availability of channels and sends it to BS through the CR nodes as shown in Fig 2.

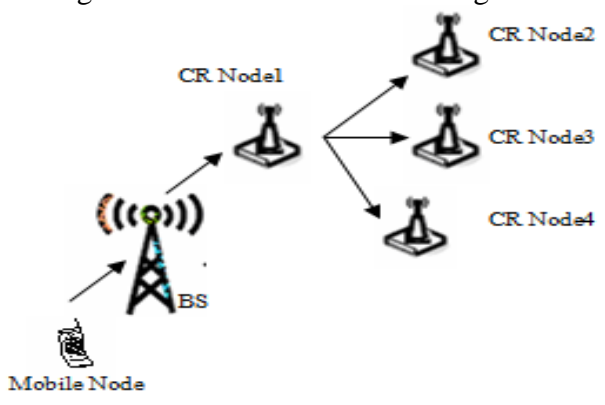

Fig 2: CR Nodes sensing the available channels

\section{CHANNEL ALLOCATION SCHEMES}

A given radio spectrum (or bandwidth) can be divided into a set of disjoint or non-interfering radio channels. All such channels can be used simultaneously while maintaining an acceptable received radio signal. The major driving factor in determining number of channels with certain quality that can be used for a given wireless spectrum is the level of received signal quality that can be achieved in each channel. 
Channel allocation schemes can be divided into a number of different categories depending on the comparison basis. For example, When channel assignment algorithms are compared based on the manner in which co-channels are separated, they can be divided into Fixed channel Allocation(FCA), Dynamic Channel Allocation(DCA) and Hybrid Channel Allocation (HCA)

In Fixed Channel Allocation (FCA) schemes, the area is partitioned in to a number of cells, and a number of channels are assigned to each cell according to some reuse pattern depending on the desired signal quality. FCA schemes are very simple, however, they do not adapt to changing traffic conditions and user distribution. In order to overcome these deficiencies of FCA Schemes, dynamic channel assignment (DCA) strategies have been introduced.

Channel Assignment Schemes can be implemented in centralized or distributed fashion. In the centralized schemes the channel is assigned by a central controller where as in distributed schemes a channel is selected either by the local Base Station (BS) of the cell from which the call is initiated or selected autonomously by the mobile. In a system with cell based control each BS keeps information about the current available channels in its vicinity. Here the channel availability information is updated by exchange of status information between BSs. Finally, in the autonomously organized distributed schemes the mobiles chooses a channel based on its local CIR measurements without the involvement of a central call assignment entity. This scheme has a much lower complexity at the cost of lower efficiency. It is important that channel assignment based on local assignment can be done for both FCA and DCA schemes.

\section{SPECTRUM SHARING}

\subsection{Basic Functionalities}

The shared nature of the wireless channel necessitates coordination of transmission attempts among CR users. In this respect, spectrum sharing provides the capability to maintain the QoS of CR users without causing interference to the primary users by coordinating multiple access of CR users as well as allocating communication resources adaptively to the changes of radio environment. Thus, spectrum sharing is performed in the middle of a communication session and within the spectrum band, and includes many functionalities of a medium access control (MAC) protocol and resource allocation in classical ad hoc networks. However, the unique characteristics of cognitive radios such as the coexistence of CR users with primary users and the wide range of available spectrum incurs substantially different challenges for spectrum sharing in $\mathrm{CR}$ ad hoc networks.

Spectrum sharing techniques are generally focused on two types of solutions, e.g., spectrum sharing inside a CR network (intra-network spectrum sharing), and between multiple coexisting CR networks (inter-network spectrum sharing) [5]. Inter-network spectrum sharing can be carried out either based on a spectrum broker that is connected to the base-station [6] [7] or in a distributed approach without support of the central network entity [8] [9].

Unlike spectrum decision, spectrum sharing mainly focuses on resource management within the same spectrum with the following functionalities:

- Resource Allocation: Based on the QoS monitoring results, CR users select the proper channels (channel allocation) and adjust their transmission power (power control) [10] [11] [12] to achieve QoS requirements as well as resource fairness.

- Spectrum Access: It enables multiple CR users to share spectrum resources by determining who will access the channel or when an user may access the channel [13]

\subsection{Proposed Architectural Concept}

The infrastructure-based network can provides sophisticated spectrum sharing method with the support of the base-station. Thus, it can exploit time slot-based scheduling and dynamic channel allocation to maximize the total network capacity as well as achieve fair resource allocation over CR nodes. Furthermore, through the synchronization in sensing operation, the transmission of CR nodes and primary users can be detected separately, which decouples sensing operation with spectrum sharing. Generally, CR networks use a periodic sensing scheme where $\mathrm{CR}$ nodes are allowed to transmit only during the transmission period followed by sensing (observation) period. In this architecture, the transmission period is synchronized over all CR nodes.

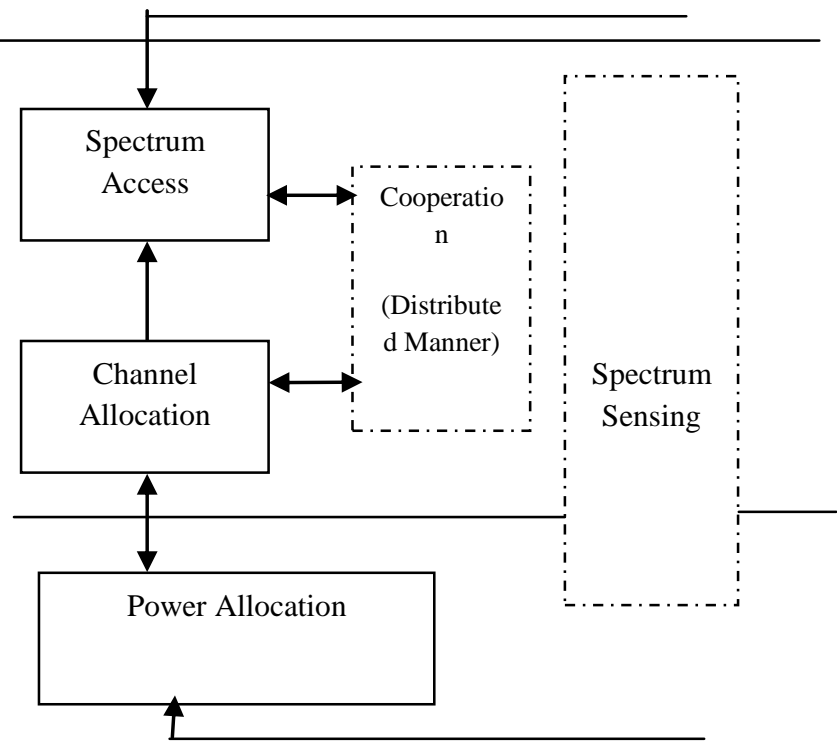

Fig 3: Functional Block diagram for Spectrum sharing CR Networks

Thus, spectrum sharing needs to focus on channel allocation or time-slot-based scheduling within this transmission period. Also spectrum sharing just exploits the spectrum availability determined in the sensing and is not directly related to spectrum sensing. Similar to spectrum sensing and decision, all sharing operations in CR users are coordinated by the basestation. Sensing and transmission intervals, determined by the sensing control in spectrum sensing, influence the performance of spectrum access. As a result, spectrum sensing should be integrated into spectrum sharing, especially in spectrum access functionality, which is shown in Fig 3.

\section{SPECTRUM SHARING FRAMEWORK FOR CR NETWORKS}

A cognitive radio is designed to be aware of and sensitive to the changes in its surrounding, which makes spectrum sensing an important requirement for the realization of cognitive radio networks. Spectrum sensing enables unlicensed users, referred to as CR users, to adapt to the environment by detecting 
unused spectrum portions without causing interference to the licensed network, referred to as the primary network.

The main objective of spectrum sensing is to provide more spectrum access opportunities to $\mathrm{CR}$ users without interference to the primary networks. Since CR networks are responsible for detecting the transmission of primary networks and avoiding interference to them, CR networks should intelligently sense the primary band to avoid missing the transmission of primary users. Thus, sensing accuracy has been considered as the most important factor to determine the performance of CR networks. Hence, the proposed research work has focused on improving sensing accuracy for interference avoidance.

CR users necessitate a periodic sensing structure where sensing and transmission operations are performed in a periodic manner with separate observation period and transmission period. In this structure, CR users should stop their transmissions during the sensing time to prevent false alarms triggered by unintended CR signals.

This periodic sensing structure introduces the following design issues:

- Interference Avoidance: Interference in CR networks depends on sensing accuracy, which is determined by the observation time. However, in periodic sensing, CR users cannot sense the spectrum bands during the transmission time, which leads to the increase in interference. Thus, for the interference avoidance, both the time in and time out need to be considered in the periodic spectrum sensing method.

- Sensing Efficiency: The main objective of CR networks is efficient spectrum utilization. Thus, the spectrum sensing functionality should provide more transmission opportunities to CR users. However, during the observation period, the transmission of CR users is not allowed, which inevitably decreases the transmission opportunities of CR users, leading to the so-called sensing efficiency issue.

\section{CHANNEL SELECTION METHOD FOR SENSING}

In the proposed work, the channel assignment scheme accounts for the interference conditions and the power constraints at different bands. Channel availability can be determined by sending service request to the BS. BS receives service request from the mobile nodes and it will send the channel request to the $\mathrm{CR}$ node. $\mathrm{CR}$ node receives the channel request and sends the broadcast message to the adjacent $\mathrm{CR}$ node. A neighbor CR node receives the broadcast message and also sends the available channel list to the BS. CR node and its neighbors, updates the channel availability list and sends response to the BS.

If $\mathrm{BS}$ receives the response from the $\mathrm{CR}$ node, it selects the available channel and sends service reply with the allocated channel to the mobile nodes. This shows the maximize utilization of a channel and it offers several services such as internet service, call service, multimedia service and so on to the mobile nodes. Our scheme, identifies 'available' channel list for each CR node. Such a list shows which channel is available to use depending on the distance among the CR node and High frequency band. Within a given neighborhood, multiple CR nodes may contend for access to one or more of the available channels.

In the proposed work, priority will be given to the primary user. It shows that if one service provider is found to be overloaded, it checks out the available channel using neighborhood CR nodes. If the service provider is found to be under-loaded, it accepts the other service providers to gain access the available channels in its region.

\section{PERFORMANCE METRICS}

In this section, we look into the performance metrics which are used to evaluate the spectrum sharing among service providers. The metrics includes block counting, service unavailability, cost, sensing efficiency and free spectrum calculation.

\section{Block count:}

The blocking probability for $\mathrm{CR}$ nodes is defined as the ratio of total blocked calls (or spectrum requests) over total calls processed by all service providers.

\section{Service unavailability (Spectral Efficiency):}

Higher Spectrum efficiency is anticipated compared to service provider systems because the blocking count of user system is lower. Thus more calls can contribute to the maximize channel utilization

3. Cost:

Within the observation time, for one service provider, the cost is determined by the number of processed calls and the holding time of each call.

4. Probability Efficiency (System Efficiency):

Probability efficiency metric for service provider is determined by the processed traffic intensity and the total traffic loaded to service provider within the observation time.

\section{Free Spectrum Calculation}

Free Spectrum will be calculated using the allocated and limited allocated spectrum values. Number of $n$ users in service providers calls in the system at time $t$ will be negligible when compared with overall performance.

\section{SIMULATION RESULTS}

In the above sections, we have seen about the sensing scheme, spectrum selection method. In this section, we present simulation results on the performance of our proposed sensing framework. Channel assignment mechanisms in the traditional multi-channel wireless networks typically select the 'Best' channel for a given transmission. In the proposed work, we are choosing the available channel with the high probability and high frequency band.

To generate utility performance measures, we assume:

1) Maximal five service providers share their spectrum and 150 nodes are chosen.

2) Maximum limit of user per channel is 10 .

3) Call arrival of each service provider is heterogeneous process.

4) Traffic rates are correlated jointly-Gaussian random variables;

5) The infrastructure of different service providers are located at the same position and the cell radii is also the same.

6) The CR nodes are present at the vertices of the cells of the service providers. 
7) Each CR node has the ability of sensing its range within the coverage limits.

8) CR nodes have the capability of detecting all the available channels that are licensed to the other service providers.

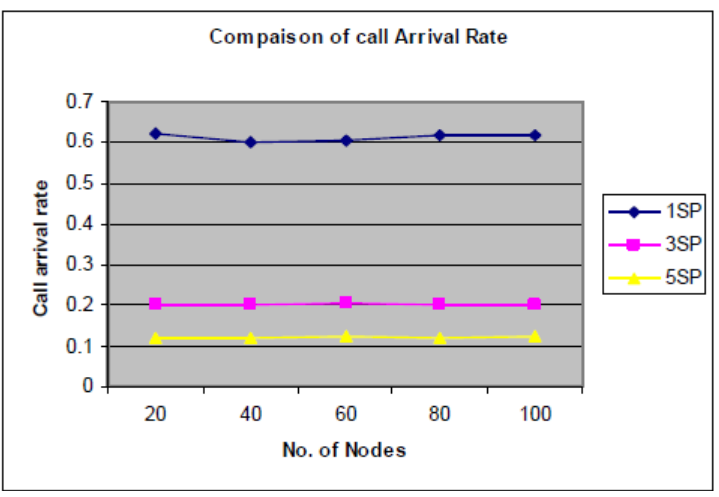

Fig 4: Comparison of Call arrival rate

We conduct simulations to verify the potential of the call arrival rate for different service providers in terms of utility performance measures. We denote call arrival rate as the number of calls in a hour. Fig 4 shows the call arrival rate for different service providers and the traffic pattern of service provider is decreased with the increased number of service providers.

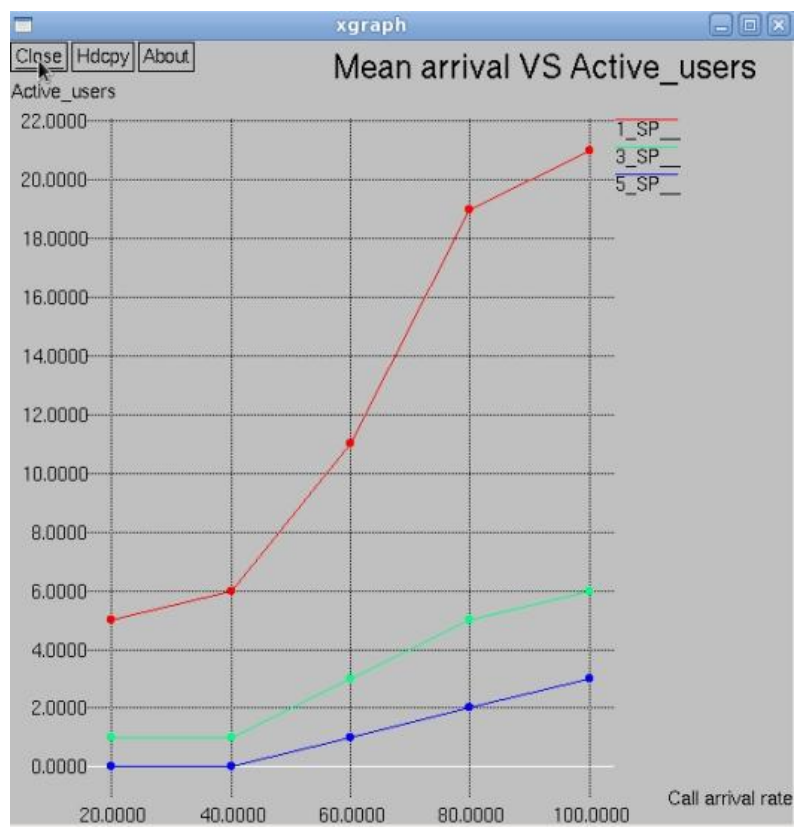

Fig 5: Mean arrival VS Active users

Fig 5 shows that, the active users are reduced when there is a higher traffic in call blocking of different service providers are highly correlated. If the call blocking is reduced among the correlated service providers then there would be increase in the active users.

Fig 6 shows that, at higher traffic rates, the call blocking rate is higher when the traffic rates of different service providers are highly correlated. The outcome is the direct result of the fact that mobile using this system can select another service provider to gain access when one service provider is overloaded.

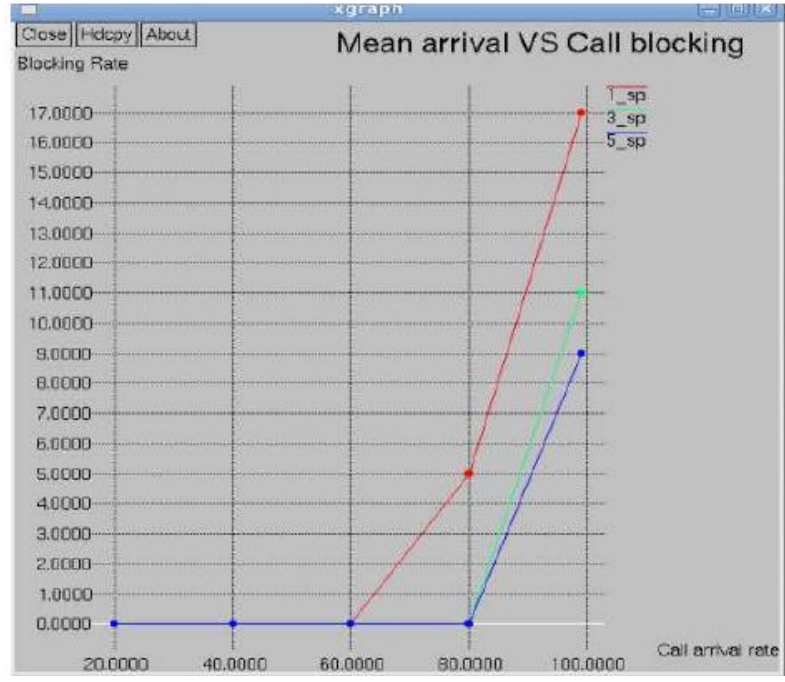

Fig 6: Mean arrival Vs Call blocking

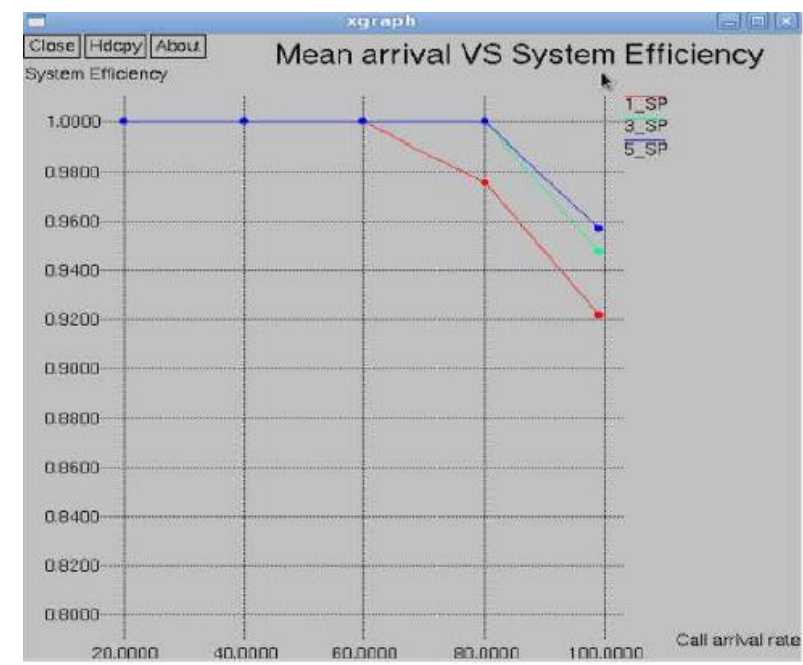

Fig 7: Mean arrival vs System Efficiency

Fig 7 shows that, at high traffic rates, the system efficiency is lower when the traffic rates of different service providers are highly correlated. When the correlation is lower, based on Fig 7, the dropped calls decrease, thus, the total processed calls increase. The system efficiency decreases when the traffic rate is beyond the system capacity. When more service providers are involved in the process of sharing, the average system efficiency is higher and the impact of number of service providers on it decreases when correlation of traffic rates are high. Thus, with five service providers the system efficiency is found at the maximum level.

\section{CONCLUSIONS}

The proposed algorithm predicts the call arrival rate of primary users and it gives an approach to predicts the call holding time of the primary users. The algorithm, which leads to the best transmission and observation time to maximize sensing efficiency satisfying the strict interference constraint of primary networks. At the higher traffic capacity, the call blocking rate is found to be high due to the higher traffic rates of different service providers are highly correlated. This prediction enhances the channel utilization of primary users and enhances the communication of primary users. CR nodes 
monitor the channel utilization of different service providers. If one service provider is found to be overloaded, it provides the information of the channel usage of the other service providers which are under-utilized. This helps to improve the channel utilization and avoids the scarce resource of the spectrum.

\section{REFERENCES}

[1] R. Kaniezhil, Dr.C. Chandrasekar, S. Nithya Rekha, 2011, "Channel Selection for Spectrum Sharing using CR Nodes" in International Proceedings of computer Science and Information Technology, ISSN: 2010-460X, vol.20, pp. 93-98, 2011.

[2] Simon Haykin, "Cognitive Radio: Brain-Empowered Wireless Communications", (Feb 2005), Life Fellow, IEEE Journal on Selected Areas in Communications, Vol. 23 No. 2, pp.201-220.

[3] Ian F. Akyildiz, Won-Yeol Lee, Kaushik R. Chowdhury, "CRAHNs: Cognitive radio ad hoc networks", (2009) Elsevier Ad Hoc Networks, pp. 810-836.

[4] V. Brik, E. Rozner, S. Banarjee, P. Bahl, "DSAP: a protocol for coordinated spectrum access in: Proc. IEEE DySPAN 2005, November 2005, pp. 611-614.

[5] Akyildiz, I. F., Lee, W.-Y., Vuran, M. C., and Mohanty, S., "Next generation / dynamic spectrum access / Cognitive radio wireless networks: a survey," September 2006, Computer Networks (Elsevier), vol. 50, pp. 21272159 .

[6] Buddhikot, M., Kolody, P., Miller, S., Ryan, K., and Evans, J., "DIMSUMNet: new directions in wireless networking using coordinated dynamic spectrum access," in Proc. IEEE WoWMoM 2005, June 2005, p. 7885.

[7] Ileri, O., Samardzija, D., and Mandayam, N. B., "Dynamic property rights spectrum access: Flexible ownership based spectrum management," in Proc. IEEE DySPAN 2007, April 2007.

[8] Lee, W.-Y. and Akyildiz, I. F., "Joint spectrum and power allocation for inter-cell spectrum sharing in cognitive radio networks," in Proc. IEEE DySPAN 2008, Chicago, IL, USA, October 2008.

[9] Lee, W.-Y. and Akyildiz, I. F., "Spectrum sharing framework for intrastructure-based cr networks," submitted for publication, 2009.
[10] Etkin, R., Parekh, A., and Tse, D., "Spectrum sharing for unlicensed bands," IEEE Journal on Selected Areas in Communications, April 2007, vol. 25, pp. 517- 526.

[11] Huang, J., Berry, R. A., and Honig, M. L., "Spectrum sharing with distributed interference compensation," in Proc. IEEE DySPAN 2005, November 2005,pp. 88-93.

[12] Zhang, L., Liang, Y., and Xin, Y., "Joint beamforming and power allocation for multiple access channels in cognitive radio networks," IEEE Journal on Selected Areas in Communications, vol. 26, january 2008, pp. 38 51.

[13] Jia, J., Zhang, Q., and Shen, X., "HC-MAC: a hardware constrained cognitive MAC for effiicient spectrum management," January 2008, IEEE Journal on Selected Areas in Communications, vol. 26, pp. 106-117.

[14] Joydeep Acharya, Roy D. Yates, "A Framework for Dynamic Spectrum Sharing between Cognitive radios", IEEE ICC 2007 proceedings, pp. 5166-5172.

[15] Wei Wang, Tiejun Lv, Zhiyuan Ren, Long Gao and Weidong Liu, "A Novel Spectrum Sharing Algorithm Based on the Throughput in Cognitive Radio Networks", IEEE, 2009.

[16] Fadel F. Digham, "Joint Power and Channel Allocation for Cognitive Radios”, IEEE WCNC 2008 proceedings, pp. 882-887.

[17] Jian Tang, Satyajayant Misra, Guoliang Xue, "Joint spectrum allocation and scheduling for fair spectrum sharing in cognitive radio wireless networks", Elsevier Computer Networks 52 (2008), pp. 2148-2158.

[18] MansoorAlicherry, RandeepBhatia, Li(Erran)Li, "Joint Channel Assignment and Routing for Throughput optimization in Multi-radioWireless Mesh Networks", MobiCom'05, August 28 - September 2, 2005, Cologne, Germany

[19] Qiwei Wang, Haitao Zheng, "Route and Spectrum Selection in Dynamic Spectrum Networks" in 3rd IEEE Proceedings of Consumer Communications and Networking Conference, 2006. CCNC 2006.

[20] R. Kaniezhil and Dr.C. Chandrasekar, "Performance Evaluation of QoS Parameters in Dynamic Spectrum Sharing for Heterogeneous Wireless Communication Networks", IJCSI International Journal of Computer Science Issues, Vol. 9, Issue 1, No 2, January 2012 ISSN (Online): 1694-0814, pp. 81-87. 